\title{
ACTUACIONES PARA MEJORAR LA SEGURIDAD Y CALIDAD $D E$ LOS EDIFICIOS
}

\author{
ALVARO GARCIA MESEGUER
}

Dr. Ingeniero de Caminos

\begin{abstract}
sinopsis
Tras aludir al origen de los fallos en construcción según estadisticas recientes y demostrar que los fallos están relacionados en proporción inversa con el grado de control ejercido en las distintas fases del proceso constructivo, el artículo se extiende sobre los conceptos de control de producción y control de recepción analizando la evolución experimentada por tales conceptos en los últimos años. Se estudian después por separado las fases de planeamiento, diseño, proyecto materiales, construcción y conservación, indicándose los puntos débiles que en cada una de ellas existen hoy día. El artículo termina presentando el concepto moderno de garantía de calidad y aludiendo a los cambios que son esperables en el futuro, como consecuencia de las nuevas ideas, en la forma de concebir y proyectar los edificios.
\end{abstract}

\section{Introducción}

Tal y como hoy se proyectan y construyen ¿son seguros los edificios, es decir, están libres y exentos de todo peligro, daño o riesgo? Desgraciadamente, los datos estadísticos de que disponemos en nuestro país sobre siniestralidad de edificios son escasos y sólo desde hace poco se ha comenzado a reunirlos (1). En cuanto a datos procedentes de otros países, las cifras no resultan concordantes. En el Reino Unido se ha estimado recientemente que el riesgo anual de muerte de una persona cualquiera, debida a un colapso estructural, es del orden de uno en siete millones (2), mientras que en Alemania Federal, Rüsch y Rackwitz ofrecen la cifra de uno en un millón (3).

En cualquier caso, el riesgo que comentamos es más reducido (3), (4) que el de muerte por explosión en viviendas ( 1 en 100.000) y el de muerte por fuego en viviendas ( 1 en 50.000), por no hablar de los riesgos de muerte por accidente de tráfico (1 en 10.000) y de muerte de un obrero de la construcción por accidente de trabajo en un determinado año ( 5 en 10.000). Todas estas cifras adquieren mayor significación a la vista del índice general de mortalidad de la población, que es del orden de magnitud del 1 por 1.000 por año a la edad de treinta años y del 6 por 1.000 a la edad de sesenta años.

Los datos anteriores parecen indicar que, hoy día, el grado de seguridad de las construcciones es razonable y cuenta con la aceptación tácita de la sociedad. Pero ello no debe ser óbice para proseguir e impulsar estudios tendentes a reducir los riesgos en la edificación, tanto a niveles teóricos como a niveles prácticos. Hay que tener en cuenta la diferente actitud de las personas ante lo que podríamos llamar riesgos evitables y riesgos inevitables. Así por ejemplo, cualquier pasajero de un avión acepta como inevitable el riesgo de que una falsa maniobra del piloto pueda conducir a una catástrofe, pero considerańa inaceptable que ese riesgo se viese incrementado, digamos en un 5 por 100, por la presencia de algún defecto mecánico en la aeronave.

Las gentes desean, con toda lógica, sentirse completamente seguras en las construcciones que les albergan, por lo que las reacciones psicológicas de la sociedad ante el hundimiento de un edificio suelen ser extremadas. La opinión pública considera el fallo de una estructura como perteneciente a la categoría de riesgo evitable y se vería muy sorprendida si las investigaciones no llegasen a demostrar la existencia de errores o negligencias en su proyecto, construcción o uso. Todo ello debe tenerse en cuenta a la hora de fijar, por las Comisiones competentes, los valores de los coeficientes de seguridad y los niveles mínimos de control que deben ponerse en práctica a lo largo del proceso constructivo.

El objeto del presente trabajo es pasar revista a las teorías y prácticas actualmente en uso dentro del marco de la seguridad estructural, señalando aquellos aspectos menos satisfactorios en los que habría que actuar para mejorar la seguridad real de los edificios, así como las últimas tendencias en la materia. 


\section{Origen de los fallos}

Los fallos de las edificaciones pueden ser clasificados en fallos últimos y fallos de servicio. En la primera categoría se comprenden aquellos que desencadenan un colapso estructural completo, lo que puede venir motivado por diversos mecanismos. En la segunda categoría se colocan aquellos fallos que, no implicando el hundimiento del edificio, lo convierten en inservible y no apto para el fin para el que fue construido, sea por exceso de deformaciones, por fisuración inadmisible, etc., sea por fallos de habitabilidad en lo que a confort se refiere (humedades, condiciones térmicas y acústicas, etc.). Dentro de los fallos de la segunda categoría conviene, a su vez, distinguir aquellos que tienen su origen en un desorden estructural, de aquellos cuyo origen radica en las instalaciones, cerramientos u obra menor del edificio.

A lo largo de este artículo y salvo expresa mención en contrario, nos referimos tan sólo a los fallos de origen estructural y, de entre ellos, en especial a los de carácter último.

En definitiva, la ruina de una estructura acaece cuando las solicitaciones actuantes superan a la capacidad resistente. Por tanto, el origen del fallo puede situarse en el uno, en el otro, o en los dos términos de la ecuación; es decir, que el fallo puede deberse ora a un aumento de las solicitaciones actuantes por encima de lo previsto, ora a una disminución de las solicitaciones resistentes por debajo de lo previsto.

Por otra parte, las construcciones atraviesan por diversas fases desde que se conciben hasta que se utilizan: planeamiento, proyecto, construcción (a partir de unos materiales y mediante aplicación de unas técnicas), uso y conservación (con eventuales cambios en el uso). En cada una de estas fases pueden cometerse errores que, por sí solos o por acumulación, pueden provocar el fallo estructural. Por ello deben analizarse por separado, conviniendo adelantar desde ahora que, según estadísticas recientes (5), un 49 por 100 de los fallos de servicio de las edificaciones tienen su origen en el proyecto, un 22 por 100 en la ejecución, un 15 por 100 en los materiales y un 9 por 100 en el uso. En cuanto a los fallos últimos, según estudios efectuados el pasado año en Suiza que abarcaron cerca de 800 fallos estructurales acaecidos después de 1971 (6), un 37 por 100 se origina en las fases de planeamiento y proyecto, un 35 por 100 en la ejecución y un 18 por 100 en las tres fases mencionadas simultáneamente.

Si se tiene en cuenta que tanto el desarrollo de las técnicas de control-de calidad en construcción a niveles teóricos, como su grado de aplicación a la realidad de la práctica, son relativamente altos en lo que se refrere a control de materiales, medios en control de ejecución y bajos o nulos en control de proyecto, es fácil derivar la conclusión de que el número de fallos en construcción está relacionado en proporción inversa con el grado de control ejercido en las distintas fases del proceso constructivo.

Ante la importancia que reviste el control de calidad en el tema en estudio, comenzaremos por hacer unas precisiones generales en torno al mismo, antes de pasar al análisis de cada una de las fases a que nos hemos referido.

\section{El control de calidad en construcción}

Hasta épocas relativamente recientes, el control de calidad en construcción se ha venido identificando con la vigilancia en obra y la realización de algunos ensayos de recepción de materiales. Sin embargo, la expresión control de calidad significa hoy día algo más elaborado, algo que lleva consigo la idea de control estadístico. La calidad, desde este punto de vista, no debe ser considerada como una cualidad abstracta sino como un parámetro cuantificable; y cuantificable no precisamente desde una óptica determinista sino desde una óptica probabilística.

Toda actividad humana en la que, a partir de unas ciertas materias primas y a través de un cierto proceso de ejecución, se llegue a elaborar un producto final, es susceptible de ser controlada estadísticamente, con tal de que el proceso se repita en unas mismas condiciones esenciales. En este sentido, cabe hablar de control de calidad de, prácticamente, cualquier cosa: desde la,fabricación de un electrodoméstico hasta la elaboración de un reportaje periodístico, pasando por el control de calidad del servicio que ofrece un hospital, pongamos por caso. En efecto, no sólo los productos manufacturados, sino también los procesos y los servicios son susceptibles de ser controlados en su calidad: todo consiste en seleccionar adecuadamente las variables que constituyan la base de seguimiento del proceso $\left({ }^{*}\right)$. Hasta la calidad de vida puede abordarse desde esta óptica y de hecho así se está haciendo hoy en algunos países.

$\left(^{*}\right)$ En el caso de hospitales a que se ha aludido, dos buenas variables base de control son: el número de reingresos que se producen por la misma enfermedad después de dada el alta (es decir, el número de altas erróneas o de falsas curaciones) y el número de enfermedades que se contraen por causas endógenas, es decir, cuyo origen radica en el propio hospital. 
Lo que sucede con la construcción es que tiene unas características propias que dificultan la adquisición y aprovechamiento de las modernas técnicas estadísticas de control de calidad. Anotemos entre ellas las siguientes:

a) El tratarse de una industria muy tradicional, tan antigua como el hombre, dotada por ello de una gran inercia.

b) El tratarse de una industria nómada, en la que la constancia de condiciones en materias primas, procesos y recursos humanos, se da con más dificultad que en otras industrias de carácter fijo.

c) El tratarse de una industria que utiliza gran cantidad de mano de obra poco cualificada. De hecho, la construcción absorbe en todos los países el empleo de personas procedentes del sector primario, que no poseen ninguna calificación profesional.

d) El tratarse de una industria que, en general, produce prototipos y no productos seriados.

e) El tratarse de una industria en la que, a diferencia de casi todas las restantes, no es aplicable la producción en cadena (productos móviles pasando por operarios fijos) sino la producción concentrada (operarios móviles actuando sobre un producto fijo).

Por todo lo anterior, sólo desde hace pocos años se están incorporando las nuevas técnicas estadísticas de control de calidad, no ya al caso particular de la prefabricación, sino también al general de las obras construidas in situ; y subsiste todavía en la mayor parte de los técnicos de la construcción una mentalidad arcaica al respecto.

\section{Control de producción y control de recepción}

El proceso de construcción puede esquematizarse como una serie de barras y nudos, tal y como indica la figura 1.

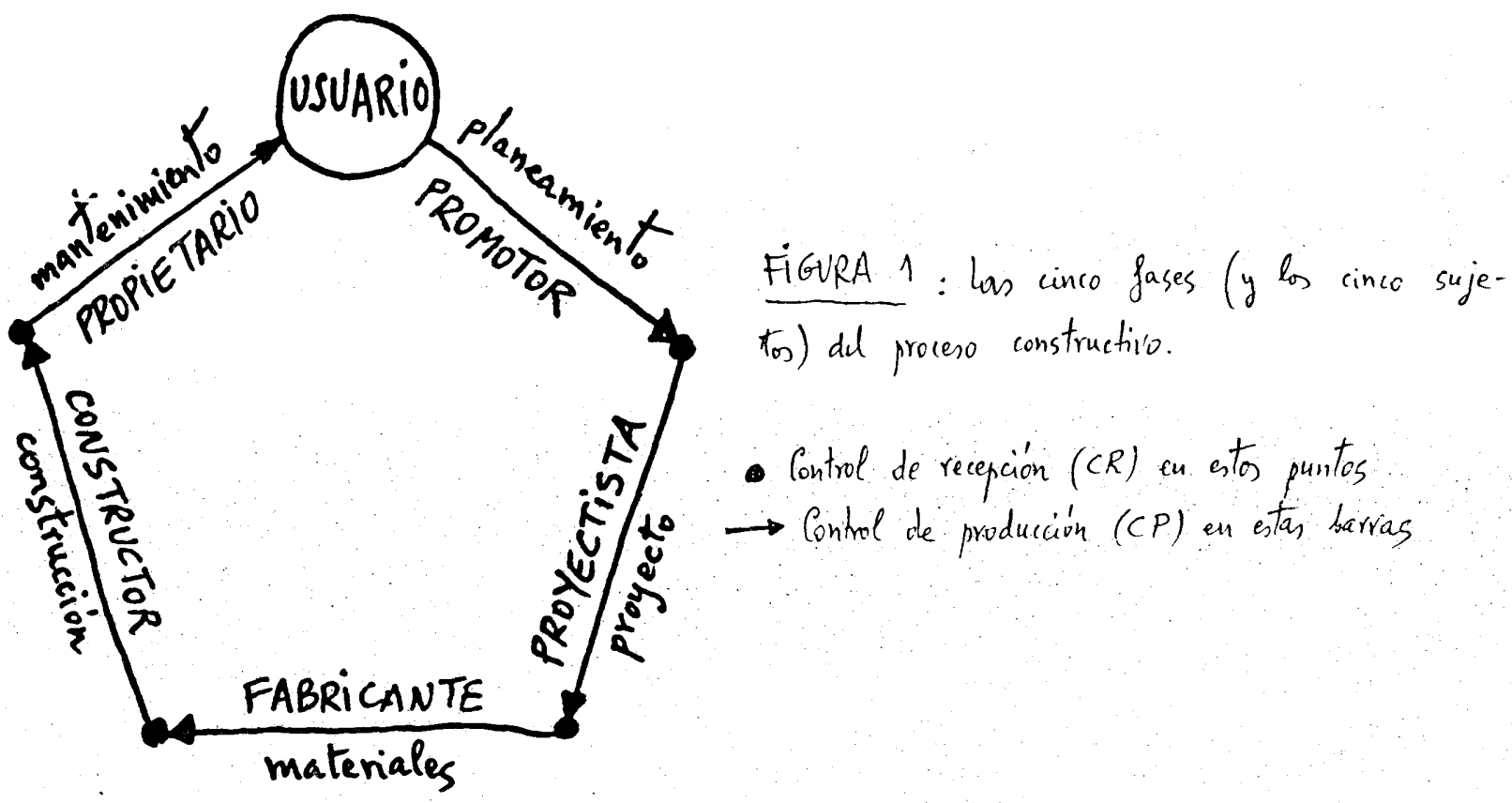

Hasta épocas recientes, la técnica de la construcción se ha venido considerando circunscrita a las tres barras inferiores, cubriendo exclusivamente los campos del proyecto, los materiales y la ejecución. Estas tres actividades llenaban por completo el contenido de las Normas e Instrucciones, las cuales solían (y suelen) dividirse en tres capítulos, dedicados precisamente a las actividades mencionadas. Pero hoy día se tiende, cada vez más, a prolongar por ambos lados el contenido técnico de la actividad de construir - las modernas Instrucciones tales como el Código Modelo CEB-FIP (7) incorporan un capítulo de conservación, precisamente como consecuencia de la importancia creciente del control de calidad- ya que la cadena de la calidad exige tener en cuenta todas las fases, arrancando y terminando en el usuario como se indica en el esquema de la figura 1. 
Al hablar del usuario no debe entenderse esta palabra en su sentido estricto (una persona física) sino en sentido amplio, ya que de alguna manera toda la colectividad es usuaria de todas las construcciones. Respecto de esta idea de carácter básico, merece la pena transcribir las siguientes consideraciones del Colegio de Ingenieros de Caminos, Canales y Puertos (8):

«La degradación de nuestro medio ambiente, la fealdad de nuestras ciudades, son testimonio inequívoco de las deficiencias del proceso edificatorio y origen del deterioro creciente en la calidad de nuestra vida ciudadana... Es necesario sensibilizar a la sociedad sobre la transcendencia que la edificación tiene en la configuración de nuestro medio ambiente, para que, superando su actual sensación de impotencia, reclame su derecho a mejorar la calidad de vida por medio de una edificación orientada hacia este objetivo...»

Como se ha dicho, la calidad es comparable a una cadena: la calidad de uso depende, en buena medida, de la calidad de la ejecución; ésta, de la calidad de los materiales y del proyecto; y la calidad del proyecto depende de la de planeamiento. La cadena no puede aislarse por tramos y debe ser contemplada en su totalidad.

¿Cómo se asegura la calidad del conjunto? A través de un doble mecanismo, articulado entre sí y constituido por dos tipos de controles: el control de producción y el control de recepción. Al conjunto de ambos se denomina control de calidad.

El contro/ de producción lo ejerce, dentro de cada barra del esquema de la figura 1, el responsable de la actividad correspondiente; se trata, pues, de un autocontrol o control interno. Por su parte, el control de recepción se ejerce en los nudos entre barras, es decir, en el paso de una actividad a otra, y lo lleva a cabo quien recibe el producto de la actividad anterior, se trata, pues, de un control externo $\left({ }^{*}\right)$.

La diferencia entre ambos tipos de controles se muestra también en sus fines y en sus medios, que no son los mismos aun cuando el objetivo final de conseguir calidad sea común. El fin del control de produccion es producir la calidad pactada al menor costo posible. El fin del control de recepción es garantizar que se recibe la calidad pactada con el menor margen de error posible. En cuanto a los medios, en el control de producción se seleccionan como variables de base para el control aquellas que mejor detecten desviaciones en el proceso y cuya medición sea rápida y barata, lo que requiere, entre otras cosas, ensayos de respuesta rápida. En cambio, en el control de recepción interesan las variables que más directamente reflejen la calidad, aun cuando su medición resulte más cara o el ensayo correspondiente sea de respuesta más lenta.

Así por ejemplo, en el caso particular del hormigón preamasado, el control de producción se basará fundamentalmente en variables tales como la relación agua/cemento, la consistencia, el contenido en cemento, etc., todas ellas de medición rápida y cuya correlación con la resistencia puede haberse establecido experimentalmente de antemano. En cambio, el control de recepción se basará fundamentalmente en la resistencia a veintiocho días, que es la variable más significativa en cuanto a calidad, aun cuando no pueda disponerse inmediatamente de los resultados de los ensayos y aun cuando estos ensayos resulten más caros que los anteriores.

El control de producción utiliza como medio más poderoso los gráficos de control, sea por variables, sea por atributos. El control de recepción, en cambio, emplea las tablas de muestreo como medio más poderoso.

En la figura 2 aparece un esquema ilustrativo de los dos tipos de control, de producción (CP) y de recepción (CR). Este esquema, aplicable a cualquiera de las fases del proceso constructivo, considera cinco aspectos sobre los que debe ejercerse control, a saber.

- medios materiales;

- medios humanos;

- materias primas;

- proceso de ejecución;

- producto terminado.

El control consiste en establecer puntos de observación en cada uno de estos aspectos (puntos cuya selección, según los casos, tiene carácter sistemático o carácter aleatorio) y analizar el resultado de las observaciones, comprobando que se ajustan a lo necesario para garantizar la constancia del proceso y el nivel de calidad del mismo.

(') Tanto en uno como en otro caso, el control puede efectuarlo directamente el sujeto interesado o puede contratarlo a un tercero (Laboratorios, Empresas u Organismos de Control). Pero esta eventual presencia de un tercero no debe confundir en cuanto a la distinción fundamental que se hace entre control de producción (propio o contratado) y control de recepción (directo o contratado). 


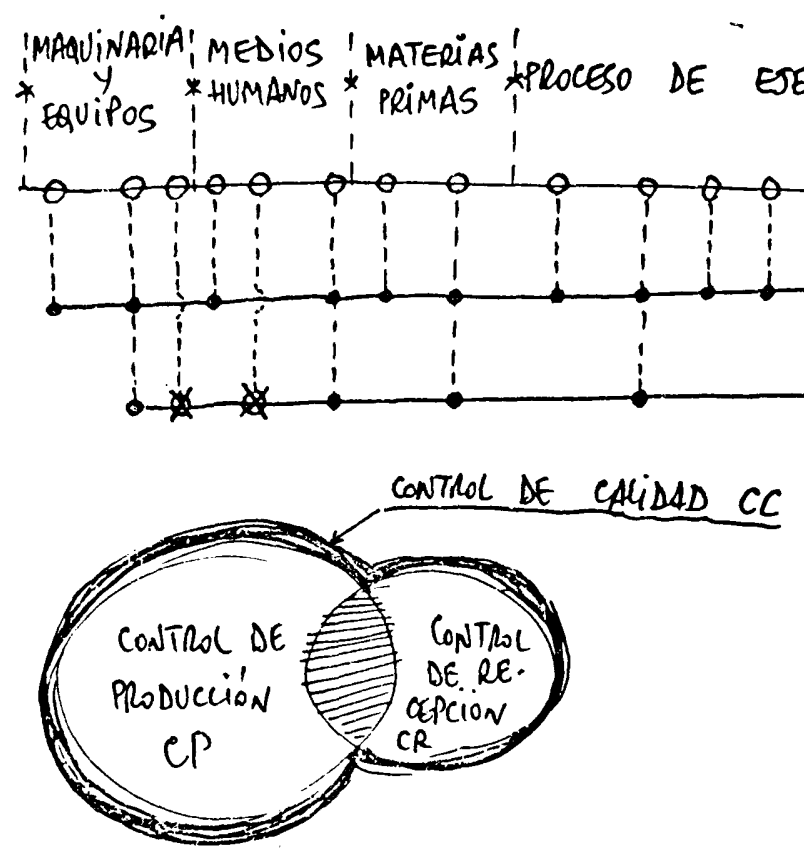

esta zona comin prede variar eritue 0 y 100 por 100

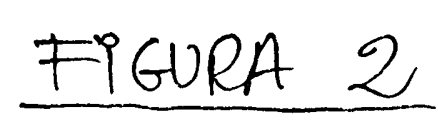

La importancis nelative del CP gel CR varian sepin los caso, dependiendo del sitema prentice. lar de Ce dil pas en custión. En situaciones limits, $C P$ ó $C R$ preden valer cero.

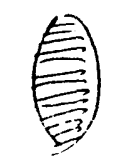

$$
\text { viner ethe } 0 \text { y }
$$

Cuando lo anterior es efectuado por el responsable de la actividad en cuestión, el resultado es un control de producción. En cuanto al control de recepción, si bien sus obsenvaciones deben concentrarse fundamentalmente sobre el producto terminado, también puede y debe incluir observaciones de los cuatro aspectos previos ya mencionados, sea aprovechando las mismas ya efectuadas y registradas en el control de producción, sea efectuándolas ex profeso, sea combinando ambas.

Por lo dicho, el CP y el CR pueden tener una zona común, como se indica en la figura 2. Esta zona común, por otra parte, puede variar entre cero y cien por cien, al igual que el CP y el CR pueden valer cero en casos límites.

Esta forma general de representar el control de calidad en construcción tiene la ventaja de que en ella quedan incluidos todos los casos posibles, no sólo dentro de un mismo país sino también entre países. Téngase en cuenta que las prácticas administrativas suelen diferir apreciablemente de país a país, lo que, unido a la amplitud de significado de la palabra control, conduce a una dificultad de entendimiento en la materia, incluso cuando son expertos los que hablan entre sí.

Una obsenvación atenta del proceso de desarrollo e implantación de las técnicas de control de calidad al campo de la construcción, conduce a constatar que suelen recorrerse las siguientes etapas $y$ precisamente por este orden:

a) No existe control de calidad.

b) Se desarrolla un control de recepción.

c) Se desarrolla un control de producción sin nexo alguno con el control de recepción.

d) Se alcanza un estadio de control de recepción más control de producción combinados, con zonas comunes.

Por otra parte y refiriéndonos ahora a las distintas fases del proceso constructivo, es un hecho que tales fases van incorporando las técnicas de control de calidad por el siguiente orden:

1. Fabricación de materiales.

2. Proceso de ejecución.

3. Proyecto.

4. Conservación y planeamiento.

Las dos secuencias cronológicas mencionadas - una relativa al modo de ejercer el control, la otra a las actividades que se controlan- constituyen una excelente pauta para conocer el grado de avance que 
un determinado país presenta en el camino de la calidad en construcción. Así por ejemplo, en nuestro país la fase 1 ha llegado al estadio $d$, la fase 2 al estadio $c$, la fase 3 comienza a entrar en el estadio $b$ y la fase 4 se encuentra en el estadio a. Otros países europeos presentan mayores grados de avance (en general, tanto mayor cuanto más septentrional es el país), encontrándose, en algunos de ellos, las fases 1,2 y 3 en el estadio d; pero ninguno ha superado hasta el momento el estadio $b$ en lo que se refiere a las fases de consenvación y planeamiento.

No deja de ser curioso (fig. 1) que el control de calidad sea tanto más fácil de implantar cuanto más alejada del usuario está la actividad correspondiente y, recíprocamente, tanto más difícil cuanto más próxima a él. El análisis de las causas de este fenómeno tendría interés desde el punto de vista tanto técnico como sociológico, pero nos llevaría muy lejos seguir ahora por ese camino.

En resumen podemos decir que, hoy día, la expresión control de calidad se aplica, en construcción, a una cualquiera de las fases o actividades del proceso constructivo considerada aisladamente de las demás, y entraña la idea de componerse de dos tipos de control diferentes y complementarios, el de producción y el de recepción. Las definiciones de estos tres controles (7) son las siguientes:

- Control de calidad. - Conjunto de acciones y decisiones tomadas para conseguir el cumplimiento de las especificaciones y asegurar posteriormente que éstas han sido satisfechas.

- Control de producción. - Conjunto de acciones y decisiones tomadas durante la producción para comprobar las operaciones del proceso y obtener una seguridad razonable de que se cumplirán las especificaciones.

- Control de recepción.-Conjunto de acciones y decisiones tomadas de acuerdo con criterios de aceptación y rechazo previamente establecidos, para comprobar el cumplimiento de las especificaciones.

\section{La fase de planeamiento}

De las cinco actividades del proceso constructivo, la de planeamiento es la que da origen y condiciona a todas las demás. En esta fase se toman, consciente o inconscientemente, decisiones que por acción u omisión van a afectar al nivel de seguridad del edificio. Y, sin embargo, no existe normalmente una conciencia clara de ello.

Las Normas e Instrucciones que regulan la seguridad estructural tan sólo se refieren a las fases de proyecto, materiales y ejecución, sin entrar en consideraciones acerca de la fase de planeamiento. Esta situación deberá cambiar en el futuro.

En efecto, las situaciones de riesgo a las que puede verse sometida una estructura pueden ser.

a) eliminadas en su propio origen;

b) evitadas mediante cambios de concepción y/o ubicación del edificio;

c) controladas a través de medidas administrativas, inspecciones, sistemas de alarma, etcétera;

d) superadas por medio de un diseño estructural adecuado;

e) aceptadas como riesgos inevitables.

Cuando se habla de seguridad estructural, los técnicos de la construcción suelen tener en mente tan sólo una de las posibilidades reseñadas, la d). Sin embargo, los aspectos a), b) y c) son fundamentales $y$, de no ser contemplados, el resultado será un aumento inevitable de los niveles de riesgo. Estas consideraciones son relevantes en fase de planeamiento, pero no han merecido hasta ahora un tratamiento sistemático en la normativa técnica de la construcción.

Para profundizar en la materia es necesario distinguir el caso del Promotor privado de aquel en que es el propio Estado, a través de sus órganos competentes, quien actúa como promotor. En el primer caso, el control del planeamiento se reduce hoy al cumplimiento de unas normas de carácter urbanístico, cuando las hay. En el segundo caso, el control de las grandes decisiones de planeamiento lo efectúa la comunidad, a través de sus órganos de representación política. Pero ni en uno ni en otro caso existen mecanismos de supenvisión que permitan detectar posibles situaciones de riesgo, a través (por ejemplo) de listas de chequeo, como forma más simple de prevención sistemática de errores de carácter aleatorio. 
Otro aspecto insatisfactorio de la fase de planeamiento es la falta de precisión con que a menudo se define el encargo al Proyectista, que es el responsable de la fase siguiente del proceso constructivo. La indefinición de una cosa suele ser causa de mala calidad de esa cosa. En tal sentido, se echa en falta la existencia de un documento que contenga normas ( 0 , al menos, directrices generales) para la definịción del encargo en los proyectos de edificación. Esas normas deberían prestar una especial atención a las condiciones de precio y plazo de los encargos, dada su influencia indirecta en la calidad del proyecto. El nivel de riesgo de aquellos edificios que han sido proyectados en un plazo perentorio es claramente superior al nivel medio, $y$ este aspecto de la cuestión afecta por igual al caso de promotores privados $\mathrm{y}$ al de la Administración como promotor $\left({ }^{*}\right)$.

Lo anterior es sólo un aspecto del problema, más general, de las relaciones entre Promotor y Proyectista, actualmente no reguladas. En el ámbito privado, cuando el segundo está ligado al primero por una relación de dependencia, no resulta posible el ejercicio de un control de recepción del encargo, lo que coloca en precario a la calidad desde su mismo origen. Análogamente puede suceder en el ámbito público, donde no es raro que se produzcan colisiones entre la ética del técnico y la del político. Sólo una normativa adecuada puede solucionar el problema, otorgando al Proyectista el suficiente respaldo en tales situaciones.

En resumen cabe decir que la fase de planeamiento es una de las menos satisfactorias, en la actualidad, desde el punto de vista del nivel de riesgo que puede generarse en dicha fase y de los mecanismos existentes para su prevención.

\section{La fase de proyecto}

Ya nos hemos referido (apartado 2) a la importancia del proyecto como origen de posibles fallos estructurales. A los datos indicados puedé añadirse que, de un estudio inglés realizado sobre hundimientos de edificios en el período 1950-1970, se deduce que un 30 por 100 de los mismos se deben a errores de proyecto (9).

Tres de los cuatro documentos de que consta el Proyecto tienen una influencia directa en la seguridad del edificio: la Memoria, donde debe justificarse la solución estructural adoptada e indicarse, a título informativo, los métodos de cálculo seguidos, el control de calidad previsto y los ensayos previos efectuados; los Planos, donde debe definirse la estructura en todo su detalle; y el Pliego de Prescripciones Técnicas Particulares, donde se especifican, con carácter de obligatoriedad, las características que han de reunir los materiales de construcción, las normas para elaboración de las distintas unidades de obra y los niveles de control exigidos para los materiales y la ejecución.

A continuación comentaremos los dos aspectos más influyentes en el tema que nos ocupa: los métodos de cálculo y el control de calidad de proyecto.

\subsection{Métodos de cálculo}

Tradicionalmente, una estructura se consideraba segura si las tensiones que aparecían en la misma, calculadas de acuerdo con alguna teoría (en general, la Teoría de la Elasticidad), no excedían de ciertos valores límites, establecidos en la Norma correspondiente. Tales valores límites, denominados tensiones admisibles, constituían por consiguiente el marchamo de seguridad de la estructura, cubriendo todas las posibles situaciones de ineptitud, desde la seguridad frente al hundimiento hasta cualquier tipo de condición de servicio. La cuantificación de esos valores límites era, normalmente, el fruto de un consenso entre los expertos más destacados de la profesión, sin una gran base racional ni científica.

Desde hace dos décadas, este planteamiento tradicional ha venido siendo desplazado por la denominada Teoría de los Estados Límites, que parte del concepto de estado límite como situación tal que, al ser rebasada, coloca a la estructura fuera de servicio. Los estados límites son clasificados en úttimos y de servicio, como ya se indicó al comienzo del apartado 2. Por otra parte, se reconoce el carácter aleatorio de todos los parámetros que intervienen en el problema, tanto los que afectan al conjunto de causas actuantes (cargas) como los que afectan al conjunto de causas resistentes (dimensiones, resistencias de los materiales). Y se postula como finalidad del cálculo la de comprobar que la probabilidad de que la estructura quede fuera de senvicio (es decir, alcance un estado límite) dentro del plazo previsto para su vida útil, se mantiene por debajo de un valor determinado que se fija a priori.

(*) Este defecto suele tener su origen, en el caso de promotores privados, en razones de economía mal entendida; y en el caso de la Administración como promotor, en razones de política mal entendida. 
La aplicación de la teoría a cada caso puede hacerse a dos niveles: el probabilista puro, en el cual no se manejan números aislados sino funciones de distribución de frecuencias; y el semiprobabilista, que, a través del empleo de coeficientes parciales de seguridad, $\gamma$, permite manejar números aislados en forma determinista. Este segundo nivel es el que desarrollan las Normas e Instrucciones de cada país y, por tanto, el que emplean prácticamente la totalidad de los profesionales en Europa.

La teoría exige, como se ha dicho, el establecimiento previo de un valor admisible para la probabilidad de que la estructura quede fuera de servicio, valor que, evidentemente, ha de ser distinto para los estados límites últimos (probabilidad de hundimiento) y para los de servicio. Los expertos han estudiado ampliamente este tema y, en el estado actual de conocimientos, han establecido para la mencionada probabilidad valores del orden de $10^{-5}$ a $10^{-6}$ para los primeros y de $10^{-3}$ a $10^{-4}$ para los segundos. Una vez fijado este valor y mediante formulaciones matemáticas complejas, es posible determinar los valores correspondientes de los coeficientes parciales de seguridad, $\gamma$, que se emplean en la práctica.

Otra forma de resolver el problema de la cuantificación de los $\gamma$ consiste en aplicar criterios de optimación económica, como el de utilidad generalizada máxima de Kjellmann o el del coste generalizado mínimo de Páez y Torroja, cuya publicación en 1952 supuso una de las primeras aportaciones internacionales en la materia (10).

Hasta aquí, la teoría. ¿Qué sucede en la práctica?

La realidad es que, partiendo de los mismos principios, cada país ha llegado a establecer un juego de coeficientes parciales de seguridad que raramente coincide entre unos y otros. Si a ello se añade la diversidad de valores numéricos con que los distintos países cuantifican las cargas y la diversidad de criterios de medición de las resistencias de materiales, se comprenderá que resulta muy difícil, por no decir imposible, efectuar una comparación de los niveles de seguridad en que se mueve cada país, no ya en la práctica de las construcciones reales, sino ni tan siquiera en la teońa de los proyectos que las definen.

A título de ejemplo y para ilustrar las diferencias mencionadas, que se describen en detalle en la referencia (11), digamos que el valor de la carga de uso que se considera en el cálculo de habitaciones de hospitales oscila entre $150 \mathrm{~kg} / \mathrm{m}^{2}$ (Noruega, Finlandia) y $400 \mathrm{~kg} / \mathrm{m}^{2}$ (Bélgica, Suecia); en escaleras de edificios de oficinas, entre $250 \mathrm{~kg} / \mathrm{m}^{2}$ (Holanda) y $500 \mathrm{~kg} / \mathrm{m}^{2}$ (Bélgica, Dinamarca, Reino Unido, Italia, Portugal); en pasillos de viviendas, entre $150 \mathrm{~kg} / \mathrm{m}^{2}$ (Dinamarca, Reino Unido, Noruega, Suecia) y $400 \mathrm{~kg} / \mathrm{m}^{2}$ (Bélgica); etc. En cuanto a la forma de medir la resistencia del material hormigón, unos países emplean probetas cilíndricas y otros probetas cúbicas, variando también, dentro del mismo tipo de probeta, la forma de curado. Respecto del acero, se emplean en Europa más de veinte tipos distintos. Etcétera.

Ante este fárrago de dificultades, la única posibilidad de obtener indicaciones comparativas acerca de los diferentes márgenes de seguridad estructural a que conduce el empleo de las distintas Normas nacionales en Europa, consiste en aplicar cada una de esas Normas a la resolución de ejemplos tipo preparados al efecto. Los ejemplos deben ser tales que, al resolverlos, se recorra la mayor parte del contenido de cada Norma, para que la comparación cubra el máximo campo posible. Esto se ha hecho ya en el seno del Comité Euro-Internacional del Hormigón, CEB (12), con la participación activa de nueve países (Bélgica, República Federal Alemana, Hungría, Comunidad Nórdica, Holanda, Polonia, Unión Soviética, España y Reino Unido) los cuales resolvieron quince ejemplos tipo utilizando sus propias Normas nacionales y el Código Modelo FIP-CEB. De esta forma, a la par que se comprobaba el grado de . utilidad del Código Modelo (nivel de claridad de exposición, grado de detalle, carácter más o menos completo de la información incluida, dudas de interpretación, etc.) se obtenía una comparación de resultados extraordinariamente útil entre las distintas normativas nacionales.

La conclusión del estudio es, hablando en términos muy generales, que el Código Modelo propuesto por el CEB presenta un nivel de seguridad que coincide bastante con la media de niveles de los países europeos; y que éstos presentan un abanico de márgenes de seguridad (con los que se está proyectando hoy día) cuyos valores extremos se colocan un 20 por 100 por encima y por debajo de ese valor medio. España se coloca en torno al 12-15 por 100 por encima, pudiendo añadirse que los países del este europeo suelen caer por debajo de la media y los del oeste por encima.

Para valorar con justeza lo anterior hay que tener en cuenta que el tema presenta dos caras, la seguridad y la economía. Por ello, las diferencias indicadas pueden enunciarse en términos de "Las estructuras de los países del Oeste son, en general, más seguras que las del Este" o en términos de "Las estructuras de los países del Este son, en general, más económicas que las del Oeste". Sería temerario, por consiguiente, emitir un juicio de valor a partir tan sólo de los datos anteriores. 
Sea como fuere, debe reconocerse que la moderna teoría de los estados límites resulta más satisfactoria que la tradicional. En efecto, gracias a la primera, el proyectista dispone hoy de una herramienta representativa de la verdadera naturaleza del problema y puede introducir en sus cálculos factores de compensación de las diversas incertidumbres que acompañarán inevitablemente a la historia de la estructura; y por su parte, los autores de Normas disponen ahora de los medios necesarios para ajustar los niveles de seguridad a las exigencias sociales del momento en cada país.

Ahora bien, hay dos aspectos del problema de la seguridad estructural que continúan sin estar resueltos a nivel internacional, a pesar de que tienen una influencia decisiva en la misma. El primero es la incidencia que el control de calidad de los materiales y del proceso de ejecución pueda tener en la seguridad estructural. El segundo es la relación existente entre seguridad estructural y fallo humano.

En lo que respecta al primer problema, unos pocos países, entre ellos el nuestro, han dado el paso de cuantificar la mencionada incidencia, pero ello ha sido sobre bases semiempíricas, no suficientemente cimentadas en estudios rigurosos de carácter científico. En lo que respecta al segundo, se trata sin duda del gran reto de nuestros días, ya que recientes estadísticas han demostrado (6) que el 75 por 100 de los fallos y el 90 por 100 de las pérdidas derivadas de fallos tienen su origen en el ser humano.

Ante esta evidencia, no podemos por menos que apoyar vivamente la opinión del canadiense Knoll (13) cuando indica que «las funciones de control resultan ser el elemento central de la prevención de fallos estructurales $y$, por consiguiente, la principal herramienta para garantizar la seguridad estructural».

\subsection{Control de calidad del proyecto}

Para una mentalidad de corte tradicional, el proyecto es el fruto de un trabajo creativo de carácter esencialmente personal y, por consiguiente, hablar de control de calidad de proyecto tiene tan poco sentido como hablar de control de calidad de un cuadro o de una escultura. Este punto de vista está siendo cada vez más sobrepasado por la realidad, ya que la creciente complejidad de las técnicas que intervienen en el proceso constructivo conduce a la necesidad de que participen en el proyecto distintos especialistas; y por otro lado, no puede negarse que una buena parte de las actividades de proyecto tienen un carácter más o menos rutinario.

Lo que sucede es que, en nuestro país, se ha sobrevalorado la figure dé proyect:sia, al amparo de unas titulaciones profesionales que venían aureoladas por un prestigio casi inítico; prestigio que se encargaban de conservar e incrementar los órganos colegiados que agrupan a estos cuerpos de élite. Esta situación, en un principio común a otros países, ha ido desapareciendo en todos ellos, pero con mayor lentitud en el nuestro.

Otros factores influyen en el asunto y explican la situación actual, claramente insatisfactoria, en la que no existe control de proyectos desde el punto de vista de su calidad. Pero no los analizaremos aquí, por haber sido ya objeto de estudios anteriores $(8,14$ y 15), limitándonos a enunciar los más importantes: falta de definición suficiente de los proyectos; espíritu de cuerpo, contrario a las colaboraciones interprofesionales; tarifas profesionales insuficientes; normativa de proyecto superabundante en unos aspectos y escasa o nula en otros; etcétera.

La seguridad y funcionalidad de los edificios mejorará notablemente el día en que la fase de proyecto reciba el mismo tratamiento que las otras fases del proceso constructivo, es decir, esté sujeta al doble juego de un control de producción y un control de recepción. En este tema pueden desempeñar un papel importante las organizaciones de control que, afortunadamente, han venido proliferando en nuestro país en la última década. La sistemática de estos controles está por definir en todo su detalle, ya que no se trata tan sólo de una supervisión del proyecto (cosa que se hace hoy en algunos sectores constructivos) sino de un verdadero control estadístico de su calidad. En esta línea se encuentran trabajando actualmente diversos grupos de expertos a nivel internacional $\left({ }^{*}\right)$ pero no son esperables resultados definitivos hasta dentro de algunos años.

Entre tanto, los Colegios profesionales de Arquitectos y de Ingenieros deberían plantearse seriamente el problema, si no quieren verse arrollados por la fuerza de unos hechos que habrán de imponerse inexorablemente, en especial el día que España se incorpore a las Comunidades Europeas.

(*) Por ejemplo, el Grupo de Trabajo "General Principles for Quality Assurance" del Comité Conjunto para la Seguridad Estructural, presidido por el profesor Ostlund; y el Grupo de Trabajo "Quality Assurance for Concrete Structures" de la Comision l del Comité Euro-Internacional del Hormigón, presidido por el autor. 


\section{Los materiales de construcción}

Ya hemos visto que, contra lo que pueda parecer a primera vista, el porcentaje de fallos estructurales debidos a defectos en los materiales de construcción es más bajo que los debidos a otras causas; $y$ hemos resaltado también que, correlativamente con lo anterior, la aplicación de técnicas de control de calidad en la fabricación y recepción de materiales es más frecuente y está más desarrollada que en otras fases del proceso constructivo. Ahora bien, el panorama que ofrecen los materiales desde el punto de vista de su fiabilidad es muy variado y abarca desde situaciones satisfactorias (caso de las barras corrugadas de acero para armar el hormigón, por ejemplo) hasta situaciones poco clarificadas por no decir abiertamente confusas. Por ello debemos comentar con algún detalle la situación y señalar los caminos de avance que parecen más prometedores.

\subsection{La certificación de la calidad de los materiales fabricados industrialmente}

Este es uno de los aspectos que más se prestan a confusión, no ya por parte de los usuarios sino hasta de los técnicos de la construcción no familiarizados con el mundo de los laboratorios de ensayo, que son los encargados de certificar la calidad de los materiales.

Hay que hacer constar, antes de seguir adelante, que la responsabilidad del empleo de los materiales viene recayendo tradicionalmente en el director de obra con carácter casi exclusivo, por imperativo del incompleto y anticuado artículo 1.591 del Código Civil. A esta situación ha contribuido el hecho de que la normativa técnica española especifica los materiales en base a normas de recepción y rara vez en base a normas de fabricación. Dicho de otro modo, se impone al director de obra la obligación de comprobar que el material reúne unos ciertos requisitos, pero no se impone (al menos, de forma clara y taxativa) al fabricante la obligación de que su material reúna tales requisitos. El resultado es un excesivo énfasis en la responsabilidad del director de obra y una difuminación de la responsabilidad del fabricante de los materiales, si bien es cierto que la jurisprudencia se viene encargando paulatinamente de corregir este estado de cosas.

Afortunadamente, existe hoy una clara tendencia a que los materiales lleguen a obra con su calidad certificada de antemano, evitando así que las obras se conviertan en inmensos laboratorios de control de recepción. Esta tendencia merece ser reforzada por todos los medios posibles. Uno de tales medios es el de difundir un conocimiento claro de las distintas posibilidades de certificación existentes, cosa que hoy no sucede a nivel general.

La pregunta es ésta: ¿Qué significado debe otorgarse a un certificado de ensayo? Para responderla, distinguiremos varios casos:

Caso A: Certificado de origen. - Se trata de un certificado que emite el propio fabricante, dando fe de que la partida del material en cuestión ha sido fabricada por él y cumple las especificaciones. La existencia de este certificado (reconocido en el Código Modelo CEB-FIP, referencia 7) supone una cierta garantía, pero añade poco a la obligación legal, tácita o expresa, que todos los materiales tienen de ajustarse a las especificaciones.

Caso B: Certificado de homologación. - Se trata de un documento emitido por la Administración (Ministerio de Industria, Ministerio de Obras Públicas y Urbanismo, etc.) que certifica que un prototipo del producto en cuestión se ajusta a lo legislado en la materia. Supone, en cierto sentido, un paso más respecto al caso $A$, ya que interviene un tercero (la Administración) en la certificación. Pero nada dice respecto a la calidad de la partida que en cada caso se suministra.

Caso C: Certificado de ensayo. - Es un documento emitido por un laboratorio en el que figuran los resultados obtenidos en el ensayo de unas muestras del material en cuestión. Para juzgar acerca del grado de garantía que supone hay que tener en cuenta las siguientes circunstancias:

- Fecha del documento: Si es reciente o se trata de un documento «histórico».

- Laboratorio de ensayo: Si está homologado o no por la Administración; si tiene o no experiencia; si es o no fiable.

- Muestras ensayadas: Si su número es o no significativo. Si fueron seleccionadas por el propio fabricante o si fueron tomadas por el laboratorio. Si se extrajeron o no al azar y de dónde exactamente.

- Ensayos realizados: Si cubren o no todos los especificados. 
El caso de mayor garantía es aquel en que un laboratorio cualificado ha tomado las muestras por sí mismo en número suficiente, las ha sometido a todos los ensayos que marca la especificación y presenta los resultados correspondientes añadiendo los valores límites especificados y un dictamen final. Pero aun en este caso, el certificado cubre tan sólo una parte de la fabricación, aquella que puede considerarse representada por las muestras ensayadas.

Importa llamar la atención sobre la variada picaresca que algunos fabricantes ponen en práctica, a base de presentar unos resultados y omitir otros, de jugar con la representatividad de las muestras, etcétera.

Caso D: Certificado (o Sello) de conformidad. - Este es el caso de mayor garantía, ya que supone una evaluación continuada de la calidad de la producción. El sistema de Sellos o Marcas de conformidad se basa en una normativa que contiene cuatro documentos:

- Especificaciones del producto.

- Normas para el autocontrol de la producción.

- Normas de inspección externa, a cargo de un Organismo independiente.

- Estatutos que regulan la concesión, uso y retirada del Sello.

En este caso, el fabricante efectúa un control continuado de su producción según reglas precisas (libros registro, gráficos de control, etc.), el cual es supervisado periódicamente por un tercero. EI Sello garantiza la calidad estadística de la producción y el fabricante continúa siendo responsable de la calidad individual de cada producto.

El sistema de Sellos de conformidad está empezando a ser potenciado en nuestro país tras diez años de experiencias que pueden considerarse piloto (16) y significa un camino de avance altamente prometedor.

Caso E: Documento de idoneidad técnica. - Es un caso análogo al anterior, pero referido a materiales no tradicionales. Goza este documento del derecho de convalidación automática entre varios países de Europa, correspondiendo su emisión en España al Instituto Eduardo Torroja. Su plazo de validez inicial es de tres años, con renovaciones anuales posteriores. Su interés es menor en el tema de la seguridad estructural por referirse, salvo excepciones, a materiales y productos que no tienen función resistente.

\subsection{Materiales no fabricados industrialmente}

Con respecto a los materiales que no se fabrican industrialmente, su control corresponde únicamente al constructor. En nuestro país se han producido fallos estructurales por mala calidad de áridos, material que cae, aunque no siempre, en este grupo. Las mejoras deben venir a través de una industrialización de este tipo de material.

Lo mismo puede decirse de los materiales que se elaboran a pie de obra, cuya responsabilidad corresponde igualmente al constructor. Es el caso del hormigón fabricado in situ, cuyas normas de elaboración y control se encuentran especificadas con todo detalle en las Instrucciones oficiales correspondientes (17) y cuyo producto base, el cemento, se elabora industrialmente. Por desgracia y a pesar de su carácter de material básico, no existe un Sello de conformidad del cemento $\left({ }^{*}\right)$, lo cual dificulta una buena ordenación del sistema de garantías en la construcción. Por otro lado, la aparición de nuevos tipos de cemento insuficientemente anunciados a los usuarios en lo que se refiere a su comportamiento diferencial respecto a los tipos tradicionales, ha creado dificultades generalizadas que, por fortuna, parecen ya casi totalmente superadas. Pero no cabe duda de que este material debería ser objeto de una mejor regulación en lo que a garantía de su calidad se refiere (no olvidemos que calidad significa, entre otras cosas, regularidad), ya que el sistema de Calidad de las Marcas por el cual se rige (alternativo del sistema de Marcas de Calidad que aquí se propugna) no ofrece completa satisfacción, dada la estructura oligopolística del mercado correspondiente. 


\section{La fase de construcción}

Aun cuando la reacción más generalizada al conocerse un fallo estructural es pensar en el constructor como primer responsable, ya hemos visto que las estadísticas demuestran que ello es así tan sólo en la cuarta parte de los casos, hablando en términos generales.

La figura del constructor ha sido analizada en muchos trabajos desde el punto de vista de la calidad $(8,14,15,18$ y 19), por lo que no nos extenderemos aquí, limitándonos a señalar cuáles son las mejores líneas de avance, en opinión del autor, para mejorar la seguridad de los edificios en lo que se refiere a la fase de construcción.

- Profesionalización y tecnificación del constructor. Debe mejorarse la normativa vigente que no exige requisitos técnicos para poder ejercer la actividad de constructor.

- Formación permanente de los técnicos y trabajadores de la construcción. Debería fomentarse a través de una legislación adecuada que exigiese a las Empresas dedicar una parte de sus recursos a la promoción y formación permanente de su personal. Hay ejemplos positivos en el extranjero.

- Calificación de oficios en la construcción. Salvo contadas excepciones, esta calificación no existe hoy. Con su implantación se conseguirían dos objetivos importantes: de un lado, la dignificación de estos profesionales, con repercusión inmediata en el nivel de calidad; de otro, el disponer de un criterio objetivo para facilitar la calificación de Empresas Constructoras.

- Fomento del autocontrol en la fase de construcción. El concepto tradicional «el constructor ejecuta y el director de obra controla» debe evolucionar cuanto antes hacia el concepto moderno «el constructor ejecuta y se autocontrola bajo la supervisión del director de obran.

De las ideas mencionadas, la última es la más importante. Hoy día se ha pasado, en muchas obras, del extremo de no ensayar nada al extremo de duplicar y aun triplicar innecesariamente ciertos ensayos. Por ello son muy positivos los pasos que en este momento se están dando, por iniciativa de SEOPAN y con la colaboración del Instituto Eduardo Torroja, hacia la implantación de un Sello de Conformidad de Empresas Constructoras (16), pasos que merecen ser reforzados y alentados.

\section{La fase de conservación}

El nivel de riesgo que para un edificio se deriva de un mal uso del mismo es, posiblemente, el más difícil de cuantificar en detalle, aunque hay datos disponibles que lo sitúan por debajo del 10 por 100 . El aumento imprudente de las cargas de uso (que puede fácilmente derivarse, por ejemplo, de un cambio de destino en el edificio), el fallo de un material resistente por falta de mantenimiento en el sistema original de protección de dicho material (corrosión de materiales metálicos, por ejemplo), son algunos de los casos de riesgos debidos al uso que han originado hundimientos en nuestro país.

La normativa técnica no se refiere, hasta ahora, a la fase de explotación y consenvación de las construcciones y éste es un defecto que debe enmendarse. Es preciso también exigir, por la vía de la ley, que a los propietarios de edificios se les entregue, junto con la obra terminada, una documentación técnica que defina completamente el producto tal y como realmente es (lo que no coincide, salvo rara excepción, con el proyecto original) y que incluya unas normas de conservación y de inspección periódica, en su caso, de determinados elementos.

Por último, deben regularse legalmente las responsabilidades del propietario y del usuario, y emprenderse campañas de divulgación al respecto. Resulta sorprendente que un bien de importancia tan inferior a la de un piso como es un automóvil, goce de una atención tan superior a la de éste en el tema que comentamos.

\section{La garantía de calidad en la edificación}

Hemos visto hasta aquí la íntima relación existente entre el nivel de riesgo y el control de calidad en las edificaciones, y hemos estudiado la situación actual de las diversas fases del proceso constructivo desde ambos puntos de vista. Procede ahora que nos preguntemos, para finalizar nuestro análisis, de qué manera podrían tenerse en cuenta, con carácter global, todos los aspectos que juegan en el problema y a través de qué mecanismos podńa asegurarse el funcionamiento correcto de todo el sistema.

La respuesta a esta pregunta se encierra en la expresión «Garantía de Calidad». En efecto, el objetivo que persigue un sistema de garantía de calidad es asegurar que todas las actividades que influyen en la seguridad de un edificio: 
- están basadas en las pertinentes condiciones fundamentales;

- se llevan a cabo de acuerdo con un plan previamente elaborado;

- se realizan con estricta adaptación a instrucciones escritas; y

- aparecen, a través de una evidencia objetivada documentalmente, como correctamente ejecutadas.

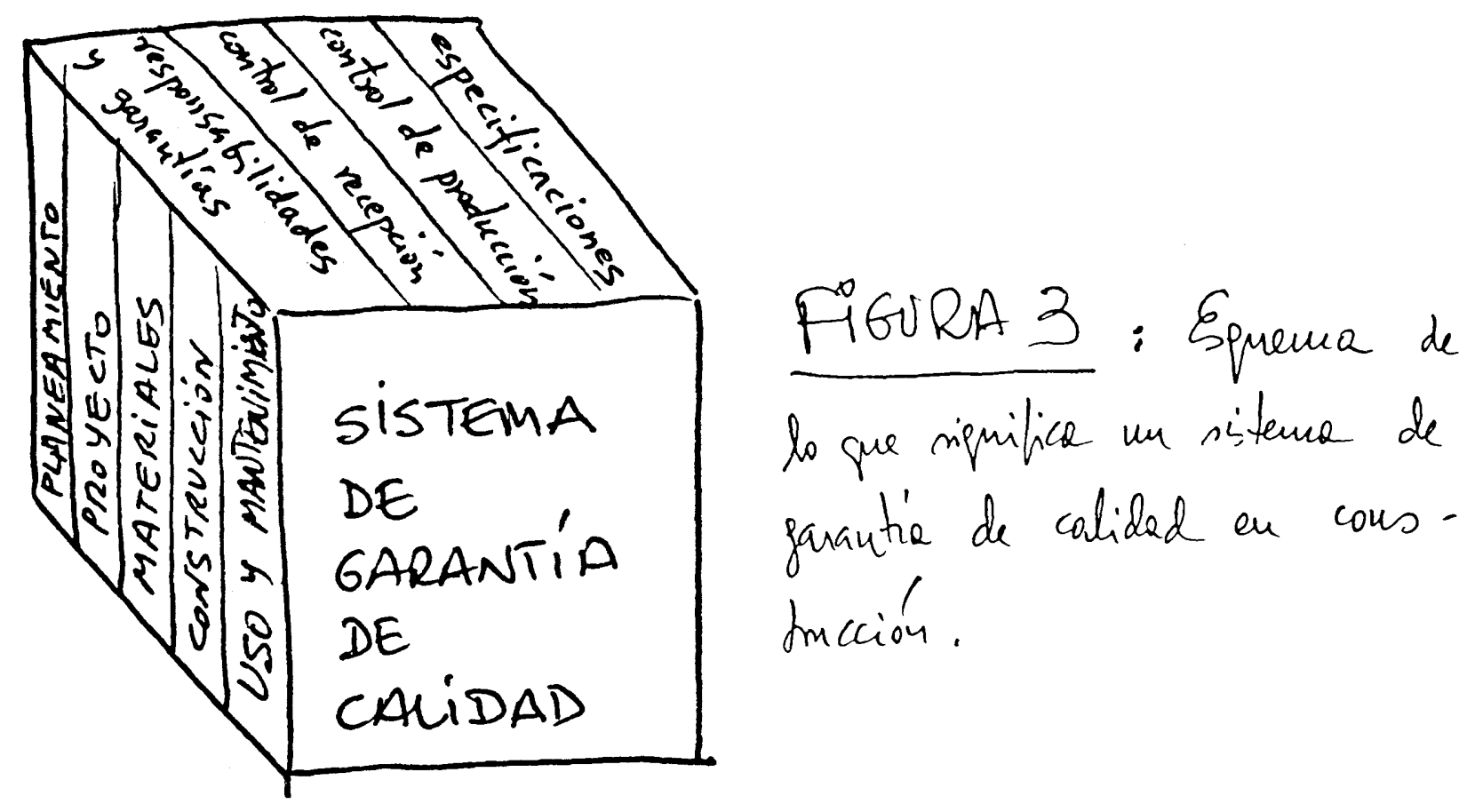

Esta definión de Garantía de Calidad, elaborada a partir de trabajos sobre la materia efectuados en la industria de la energía nuclear (20), ha sido adoptada, a propuesta del autor, por el Grupo Editorial del Joint Committee on Structural Safety (21) y aparece ilustrada en el esquema de la Figura 3.

Hay que suponer que la aplicación del moderno concepto de Garantía de Calidad al sector de la construcción traerá consigo un cambio en las formas de proyectar y construir al uso, en el sentido de ampliar el abanico de contingencias que hasta ahora se venía manejando, con énfasis especial en lo relativo a posibles fallos humanos. La verificación de la seguridad estructural, construida hasta ahora sobre bases puramente numéricas que podían condensarse en la expresión:

$$
\text { cargas } \leq \text { resistencias }
$$

pasará dentro de algunos años a formularse sobre bases más cualitativas y constituirá una parte tan sólo de todo un Plan de Seguridad específico para cada edificio que se haya de construir.

Veamos qué significa esto, en líneas muy generales, de la mano de los últimos trabajos suizos sobre la materia (4).

\subsection{El Plan de Seguridad}

Para salvaguardar la seguridad de los usuarios, de los trabajadores de la construcción y de terceras personas, hay que prestar atención desde el origen mismo de un proyecto a todas las Situaciones de Riesgo («Hazard Scenarios») que pueden presentarse durante el proceso constructivo, tales como:

a) Planeamiento, proyecto, construcción o uso de la estructura fuera de control, por ejemplo:

- superación de valores previstos para las cargas;

- no alcanzarse las resistencias previstas para los materiales:

- accidentes locales que conduzcan a una de las dos circunstancias anteriores;

- falta de mantenimiento.

b) Efecto de condiciones climáticas extremas de cualquier tipo, tales como:

- viento, agua, hielo, nieve, temperatura, etcétera;

- efectos combinados. 
c) Efectos de deterioro en el tiempo, fenómenos de fatiga y cansancio, etcétera, que pueden repercutir en una baja de resistencia estructural.

d) Efectos de la subpresión del agua, del agua corriente, de influencias geológicas o tectónicas, incluyendo los efectos de las construcciones vecinas reales o potenciales.

Las Situaciones de Riesgo deben describirse en un Plan de Seguridad, el cual deberá ser preparado en la fase de planeamiento por el Director del Proyecto, en íntima conexión con el Propietario y convenientemente asesorado por especialistas. Este Plan debe especificar qué medidas habrán de adoptarse para contrarrestar las situaciones de riesgo, medidas que, básicamente, pueden consistir, como ya se dijo en el apartado 5, en:

- eliminar las situaciones de riesgo en su propio origen;

- evitarlas mediante cambios de concepción y/o ubicación del edificio;

- controlarlas a través de inspecciones, sistemas de vigilancia, sistemas de alarma, etcétera;

- superarlas mediante un diseño estructural adecuado;

- aceptarlas como riesgos inevitables.

El Plan de Seguridad especificará en particular aquellas situaciones de riesgo que deben ser cubiertas por el proyectista mediante una apropiada capacidad resistente de la estructura. Parte de este Plan estará formado por un plan de control de datos que incluirá las comprobaciones necesarias, medidas de supervisión y procedimientos de chequeo.

En el momento en que el edificio es entregado al Propietario, éste toma sobra sí la responsabilidad de su uso y mantenimiento. Para facilitarle esta tarea, el Director del Proyecto le entregará toda la información escrita necesaria.

Cuando el Plan de Seguridad mencione riesgos específicos como inevitables, habrán de detallarse las medidas necesarias para librar de peligro a las personas, a la vez que se definen las responsabilidades económicas correspondientes a tales eventualidades.

\subsection{Sistemas de prevención}

¿Cómo asegurarse de que todo lo anterior funcionará correctamente?

Las respuestas posibles a esta pregunta son, en el extremo, solamente dos: o lo asegura la Administración del Estado o lo asegura la iniciativa privada. Se trata en definitiva de una cuestión de carácter político en la que es determinante la forma en que cada sociedad se encuentra organizada. Por consiguiente, la respuesta corresponde a cada país.

Este tema, verdaderamente transcendental, constituyó el objeto de estudio del II Coloquio Europeo, de la EOOC (European Organisation for Quality Control) sobre "Garantías en la Edificación», celebrado en Madrid en noviembre de 1979. En dicho Coloquio se pasó revista a los distintos modelos existentes en Europa, desde el sistema francés de seguro obligatorio en construcción hasta el sistema inglés de acción independiente del seguro y la Administración. En cualquier caso, el congreso proclamó unánimemente que, cualquiera que sea el sistema escogido, el empleo de un mecanismo de seguro supone un eficaz instrumento.

En efecto, un seguro de daños en construcción cuyo beneficiario sea precisamente el usuario de la vivienda, significaría para éste la solución inmediata a muchos problemas que hoy le preocupan: mal funcionamiento de las instalaciones, humedades, fallos de confort.. En todos estos casos de fallos de habitabilidad, la Compañía se hańa cargo inmediato de las reparaciones y repercutináa después en el sujeto responsable. En otro orden de cosas y caso de fallos últimos, el seguro debería suponer el pago inmediato de indemnizaciones a los familiares de las víctimas, cosa que hoy no sucede $\left({ }^{*}\right)$. En fin, la intervención de una Compañía de Seguros desde el mismo origen del proceso edificatorio clarificaría el panorama y crearía una valorización automática de la calidad en las distintas fases del proceso, ya que el nivel de las tarifas se adecuaría a cada caso; y el control de calidad, hoy inexistente en tantas ocasiones, se impondría por sí mismo.

Hemos llegado así al final de este trabajo, una de cuyas intenciones es la de ofrecer un panorama de base a las Compañías de Seguros, tratando de llamar su atención sobre la gran tarea que aún queda por desarrollar en la prevención de riesgos en construcción.

(*) En el último juicio por fallo estructural al que el autor hubo de asistir como perito, las viudas de los obreros fallecidos en el accidente no habian percibido ninguna cantidad de dinero cinco años después de la muerte de sus maridos. 
(1) Grupo de Trabajo: «Patología de la Construcción», del Comité de Construcción de la Asociación Española para el Control de la Calidad. Informe interior.

(2) Construction Industry Research and Information Association: «Rationalisation of safety and serviceability factors in Structural Codes». Informe núm. 63. Londres, 1978.

(3) H. Rüsch y R. Rackwitz: «The significance of the concept of probability of failure as applied to the theory of structural safety»). Edición especial de la publicación conmemorativa titulada «Desarrollo, diseño y construcción», con motivo del centenario de Held-Francke Banaktiengesellschaft, Munich.

(4) W. Bosshard: «Structural Safety, a matter of decision and control». Informe S-9/79 de la serie IABSE Surveys, Zurich 1979.

(5) J. Calavera: «Riesgos de ruina derivados de los materiales y de la ejecución». Ponencia presentada en el II Coloquio Europeo de la EOQC sobre «Garantías en la Edificacion». Madrid, noviembre 1979.

(6) J. Schneider. Informe presentado al Joint Committee on Structural Safety en su reunión de 7 de mayo de 1979 en Zurich.

(7) Comité Euro-International du Béton: «Code Modele CEB-FIP pour les structures en béton». Bulletin d'information núm. 124/125 del CEB, París, abril 1978.

(8) Colegio Nacional de Ingenieros de Caminos, Canales y Puertos: «Declaración sobre la Edificación». Madrid, noviembre, 1978.

(9) L C. P. Yam: «Anatomy of structural safety an a Who does What guide to quality assurance». Building Research Station, Londres, 1979.

(10) Alfredo Páez y Eduardo Torroja: «La determinación del coeficiente de seguridad en las distintas obras». Editado por el Instituto Técnico de la Construcción y del Cemento, Madrid, 1952.

(11) Manfred Stiller. «Règles normalisèes pour les constructions, sur le plan européen. Désir et réalité». Versión francesa del original alemán, publicada por la Asociation Française, du Béton, Pańs, marzo 1974.

(12) Comité Euro-International del Hormigón: «Trial and comparaison calculations». Boletin de información núm. 129, París, octubre 1978.

(13) Franz Knoll: «Safety building codes and human reality». Comunicación presentada al XI Congreso de la Internacional Association for Bridge and Structural Engineering. Viena, septiembre 1980.

(14) Comité de Construcción de la Asociación Española para el Control de la Calidad: «Análisis de la situacción actual del control de calidad de la construcción en Españan. Madrid, julio 1971.

(15) Ibidem: «Recomendaciones de actuación para mejorar la calidad de la construcción en España». Madrid, junio 1973.

(16) Comité Promotor de las «Primeras Jornadas de Calidad en la Construcción a través de las Marcas y Sellos». Documento de Trabajo (45 páginas) editado en Madrid, 1979.

(17) Comisión Permanente Interministerial del Hormigón: «Instrucciones EH73 y EP77 para obras de hormigón en masa, armado y pretensado». Ministerio de Obras Públicas y Urbanismo, Madrid, 1973 y 1977.

(18) Dirección General de Arquitectura y Vivienda, del MOPU: «Libro Blanco sobre la Edificación». Madrid, julio 1978.

(19) Colegio Oficial de Ingenieros de Caminos, Canales y Puertos: «Boletín de información núm. 24 dedicado a la calidad en la edificación» (contiene 56 referencias bibliográficas). Madrid, diciembre 1978.

(20) José Sancho Jimeno: "Quality Assurance. Suggestions for a more precise definition or terms used". Comunicación presentada a la XXIII Conferencia de la European Organisation for Quality Control. Budapest, septiembre 1979.

(21) L Ostlund: «Second draft on General Principles of Quality Assurance for structures». Comunicación interior del Editorial Group del Joint Committee on Structural Safety, Lund, diciembre 1979. 


\author{
Alvaro García Meseguer, \\ Dr. Ingénieur des Ponts et Chaussées
}

L'auteur de cet article, après avoir évoqué l'origine des défauts dans la construction suivant des statistiques récentes et montré que les defaillances sont inversement proportionnelles au degré de contrôle exercé dans les différentes phases du processus constructif, porte sur les concepts de contrôle de production et de contrôle de réception et analyse l'évolution de ces concepts au cours des dernières années. Puis, il fait une étude de chacune des phases de conception, projet, matériaux, construction et entretien, indiquant les points faibles existant actuellement dans chaque phase. Finalement, l'auteur présente le concept moderne de garantie de qualité et fait allusion aux changements qui sont à espérer à l'avenir, par suite des nouvelles idées, dans la manière de concevoir et de faire les projets des bâtiments.

\title{
SUMMARY
}

ACTIONS TO IMPROVE BUILDING SAFETY AND QUALITY

\begin{abstract}
Alvaro García Meseguer,
\end{abstract}
Dr. in Civil Engineering

After discussing the most recent statistic on construction failures and proving that such failures occur in inverse proportion to the level of supervision applied during the various stages of the construction process, the article enters into the subjects of production control and receiving control, analysing the evolution of both concepts in recent times. The design, materials selection, construction and maintenance stages are individually studied with indication of the weak points currently recognized in each stage. The article ends with the presentation of the modern quality assurance concept and describing the changes that can be expected in the future as the result of the new ideas in the way buildings are conceived and designed.

\section{ZUSAMMENFASSUNG}

MASSNAHMEN ZUR VERBESSERUNG DER SICHERHEIT UND QUALITAET VON GEBAEUDEN

Dr. Alvaro García Meseguer,

Ingenieur

Nachdem auf den Ursprung der Mängel im Bauwesen gemäss den letzten Statistiken hingewiesen und eine Beschreibung darüber gegeben wird, dass die Mängel in umgekehrten Verhältnis zu den Kontrollmassnahmen während der verschiedenen Bauphasen stehen, geht der Artikel auf die Konzepte der Wualitätskontrolle bei der Herstellung und Abnahme ein und untersucht die Entwicklung dieser Konzepte in den letzten Jahren. Es werden im Anschluss gesondert die Planungs-, Entwurfsund Projektphasen, die Materialien, der Bau und die Wartung studiert und dabei die zur Zeit in jedem einzelnen Gebiet bestehenden schwachen Punkte aufgezeigt. Abschliessend verweist der Artikel auf das moderne Konzept der Gütekontrolle und auf die in Zukunft zu enwartenden Veränderungen als Folge der neuen Ideen bei der Auffassung und Planung der Gebäude. 